\title{
Evaluation of a routine follow-up visit after an internal fixation of proximal femoral fracture
}

\section{Söderlund, Tim P.}

2017-02

Söderlund, T P \& Kuorikoski , J M 2017 , ' Evaluation of a routine follow-up visit after an internal fixation of proximal femoral fracture ' , Injury , vol. 48 , no. 2 , pp. 432-435 . https://doi.org/10.1016/j.injury.20

http://hdl.handle.net/10138/236599

https://doi.org/10.1016/j.injury.2016.12.020

publishedVersion

Downloaded from Helda, University of Helsinki institutional repository.

This is an electronic reprint of the original article.

This reprint may differ from the original in pagination and typographic detail.

Please cite the original version. 


\title{
Evaluation of a routine follow-up visit after an internal fixation of proximal femoral fracture
}

\author{
Joonas MM. Kuorikoski ${ }^{\mathrm{a}, \mathrm{b}}$, Tim P. Söderlund ${ }^{\mathrm{a}, \mathrm{b}, *}$ \\ a Trauma unit, Department of Orthopaedics and Traumatology, Töölö Hospital, Helsinki, Finland \\ ${ }^{\mathrm{b}}$ Academic Medical Centre Helsinki, Helsinki, Finland
}

\section{A R T I C L E I N F O}

\section{Article history:}

Accepted 21 December 2016

\section{Keywords:}

Hip fracture

Proximal femur fracture

Control visit

Follow-up visit

Outpatient visit

Internal fixation

Complication

\begin{abstract}
A B S T R A C T
Background and purpose: Post-operative follow-up after internal fixation of fractures is a common practice. The goal of this study was to evaluate the necessity of a routine follow-up visit after internal fixation of a proximal femoral fracture. Our hypothesis is that these follow-up visits do not result in a change in the treatment plan, but add an extra cost to the health care system and lead to the purposeless utilisation of limited resources.

Patients and methods: A retrospective study of 428 consecutive patients ( 431 fractures) with a scheduled outpatient clinic visit after internal fixation of proximal femoral fractures in a single hospital during years 2012-2013. We noted any changes in the patients' treatment plans based on the first follow-up visits, including scheduled visits up to ten weeks after internal fixation.

Results: None of the patients showed signs of infection, implant failure or loss of reduction requiring reoperation at the scheduled follow-up visit. In only one $(0.23 \%)$ visit a change in treatment plan was made as a result of the scheduled follow-up visit (decision to remove the distal screws from the long intramedullary nail to obtain dynamic compression). Scheduled visits did not occur for the following reasons, death (11.8\%), visit to emergency department prior scheduled visit (3.2\%), and not known (3.9\%). Conclusions: The first scheduled visit within ten weeks after internal fixation of a proximal femoral fracture leads to no changes in treatment. We recommend considering the need of these follow-up visits.

(C) 2017 Elsevier Ltd. All rights reserved.
\end{abstract}

\section{Introduction}

The health care expenses were 2013 in Finland were 9.5\% of the nations gross domestic product which was slightly higher than the average of OECD countries ( $8.9 \%$ ), but significantly lower than in US (17.4\% in 2009) [1] Also, the health care expenses have increased from 2000 to 2014 [1]. The Choosing Wisely Initiative aims for the reducing of unnecessary costs in health care by naming tests, interventions or services which are overused or unnecessary [2]. A routine post-operative visits after third molar removal under intravenous sedation was not necessary as shown in a randomized clinical trial [3]. In a primary total hip arthroplasty with prostheses with excellent long-term results, a routine follow-up of asymptomatic patients is not necessary [4]. In the light of the above it is necessary to evaluate our common practices in orthopaedic trauma as well.

\footnotetext{
* Corresponding author at: Department of Orthopaedics and Traumatology Helsinki University Hospital Topeliuksenkatu 500260 Helsinki, Finland.

E-mail address: tim.soderlund@helsinki.fi (T.P. Söderlund).
}

The estimated global incidence of proximal femoral fracture (PFF) in 1990 was 1.31 million, with a prevalence 4.48 million and 0.7 million deaths [5]. Also, the number of hip fractures globally is estimated to increase to 2.6 million by 2025 and to 4.5 million by 2050 [6]. As a consequence the socio-economic impact of these fractures can be expected to increase significantly [6]. According to the National Institute for Health and Welfare (THL) in Finland, every year about 7000 PFFs occur in Finland. The average health care expenses in 2005 in Finland for the first year after proximal femoral fracture were around $19000 €[7]$.

Routine radiographs in the first post-operative visit are common in orthopaedic practice. For example, the AO Foundation recommends routine $\mathrm{x}$-rays six weeks after the internal fixation of PFF and subsequently every-six weeks until the fracture has healed [8]. However, the efficacy of these routine follow-ups is unclear and the effect of these radiographs on treatment has been challenged. In a recent study of 200 acute fractures, only one routine radiograph during the first post-operative visit led to a change in the therapy [9]. This change in the radiograph led to no changes in treatment. Some evidence indicates that early radiographs after ankle fracture fixation do not lower the complication 
rate and could be omitted [10]. In only three of 878 ankle fracture patients $(0.3 \%)$, the treatment plan was changed in the first followup visit due to radiological findings [11]. Stone et al. [12] demonstrated that post-operative radiographs taken two weeks following plate fixation of distal radial fractures rarely resulted in changes in patient management, but added an extra cost to the health care system [12]. Thus, although the benefit of early postoperative radiographs remains questionable, they do increase costs for the patient and health care system. Thus the need of routine post-operative follow-ups with radiographs should be evaluated.

In this study, we assessed whether the first scheduled follow-up visit after internal fixation of a PFF alters treatment. Our hypothesis is that first post-operative follow-up visits, scheduled up to ten weeks post-operatively, result in no changes in the treatment plan, but only adds an extra cost to the health care system and lead to waste of limited resources.

\section{Patients and methods}

This is a retrospective study of consecutive operatively treated proximal femur fracture patients scheduled for post-operative follow-up in Töölö Hospital (part of the Helsinki University Central Hospital in Helsinki, Finland). Töölö Hospital is the only public orthopaedic trauma hospital in Helsinki (population 630000 inhabitants) and it serves as a tertiary referral hospital for approximately 1.8 million inhabitants. The inclusion criteria for the study were: 1) Proximal femoral fracture treated with internal fixation in our hospital between 1 January 2012 and 31 December 2013 and, 2) scheduled post-operative follow-up according to our hospital protocol. The exclusion criteria were: 1) PFF treated with hemi- or total arthroplasty, 2) patients outside our hospital district (follow-up at other institutes), 3) patients with femoral shaft fracture (with no ipsilateral proximal femoral fracture), 4) patients who underwent prophylactic surgeries due to primary bone malignancies or metastatic changes in the bone, 5) pathological fractures (metastatic changes or primary tumors).

If a patient had bilateral proximal femoral fractures, the fractures were counted as two individual fractures. We used Nordic Classification of Surgical Procedures (NCSP) codes NFJ50, NFJ52, NFJ54, and NFJ60 to identify patients possibly eligible for the study.

The operative and post-operative treatment protocols were standardized and remained the same throughout the study period. Open reduction and internal fixation was done according to AOprinciples. Weight-bearing was restricted only in younger patients with high-energy neck of femur fracture (NOF). At discharge all the patients were instructed to contact our hospital in case of any problems with the operated hip. Our hospital protocol included scheduled visit to outpatient clinic at 6 and 12 weeks after the index surgery. Due to the limited resources the 6 weeks follow-up visit was usually within 4-9 weeks. We decided to include first visits up to ten weeks because the last routine follow-up was never this early in our practice. The scheduled visit included a physical examination and routine radiographs of the hip (anteroposterior and lateral projections).

We collected data from electronic medical records, radiographs and radiograph reports. For each patient we collected age, fracture type, method of internal fixation, time from surgery to the followup visit, and any possible change in the patient's treatment plan at the scheduled visit. Methods of internal fixation included cannulated screws, dynamic hip screw, short intramedullary nail, long intramedullary nail and other (plating or a combination of several methods). Changes in treatment plan included any alteration of the treatment plan that deviated from normal post-operative management. The changes were: antibiotics due to a wound infection, and re-operation for any reason (infection, hardware failure, loss of fracture reduction). The number of visits is calculated on by the number of scheduled visits (e.g. by the number fractures), and not by the number of patients. We used IBM SPSS Statistics for Windows, Version 21.0 (Armonk, NY, USA: IBM Corp) to analyse the data and the Wilcoxon Rank-Sum Test with an $\alpha$-value of less than $5 \%$ (0.05) for statistical significance. This was a retrospective study of medical records, so Finnish law does not require approval by an ethics board. The research council of the Helsinki University Central Hospital approved the study protocol.

\section{Results}

During the study period ( 2 years) a total of 428 consecutive PFF patients with 431 fractures were treated with internal fixation and scheduled to follow-up at our outpatient clinic. The female-tomale ratio for fractures was approximately $2: 1$ (66.2\% vs. $33.6 \%$, 286 vs. 145 fractures) with a mean age of $74.2 \pm 17.4$ years. The average age of the men was $65.0 \pm 18.0$ years (range $20-101$ years), which was significantly lower than the average age of the women, which was $78.9 \pm 15.0$ years (range $18-101$ years) $(p<0.001)$.

The majority of the fractures $(n=312,72.4 \%)$ were trochanteric, followed by femoral neck $(n=76,17.6 \%)$ and subtrochanteric fractures $(n=43,10.0 \%)$. The mean age of the patients with femoral neck fractures treated with cannulated screws or DHS was $60.8 \pm 17.6$ years compared to $77.9 \pm 15.4$ years for the patients with trochanteric or subtrochanteric fractures $(p<0.001)$. DHS was the most common internal fixation method on neck of femur fractures, used in $56.6 \%$ (43 of 76) of cases. Short intramedullary nail $(48.4 \%, 151$ of 312$)$ was the most common method for fixation of pertrochanteric fractures. Subtrochanteric fractures were mainly fixed by long intramedullary nail ( $86 \%, 37$ of 43$)$.

For the study population 431 post-operative follow-ups were scheduled to outpatient clinic. Majority of the patients $(n=349$, $81.0 \%$ ) showed up to the scheduled visit. At the first post-operative visit, radiographs were taken of all patients. None of the patients showed implant failure or loss of reduction requiring re-operation. None of the patients showed signs of infection. In only one patient treatment plan was changed in the follow-up visit. This patient (age 39 years) had the distal screws removed from the long intramedullary nail in order to obtain dynamic compression for the subtrochanteric fracture. Thus the scheduled visit led to a change in $0.23 \%$ of patients coming to the scheduled visit. The type of fracture and method of internal fixation of the patients attending to scheduled follow-up appear in Table 1.

Fourteen patients (3.2\% of all scheduled visits) had a sixteen unplanned visit to ER before the scheduled routine outpatient clinic visit. The reasons for unplanned visits were (percentages calculated from all scheduled visits): deep wound infection requiring intravenous antibiotics and revision surgery $(n=3$, $0.7 \%)$, new fracture on the contralateral femur $(n=3,0.7 \%)$, periprothetic fracture or implant failure $(n=3,0.7 \%)$, suspicion of superficial wound infection ( $n=2,0.5 \%$ ), pain in the operated leg ( $n=2,0.5 \%)$, wound haematoma $(n=1,0.3 \%)$, urinary tract infection $(n=1,0.3 \%)$, and fall without new fractures $(n=1,0.3 \%)$. The unplanned visit led to deviation from normal post-operative management in eleven of these sixteen visits (69\%).

Fifty-one patients (11.9\% of scheduled visits) died before the scheduled post-operative visit. The average age of these patients was $84.9 \pm 11.3$ years and $60.8 \%$ were females. Seventeen patients (4.0\%) did not show up to their scheduled visits for unknown reasons. Revision surgery for post-operative infections and reoperations for these fractures is done in our hospital for patients in our hospital district. Therefore it is unlikely that infection or revision surgery would have been the reason for missing the scheduled visit. The electronic medical records showed that these patients were not operated in other public hospitals either. The 
Table 1

Types of proximal femoral fractures and methods of internal fixation in 349 patients attending the scheduled follow-up visit.

\begin{tabular}{|c|c|c|c|c|c|c|}
\hline & & \multicolumn{4}{|c|}{ Fracture Type } & \multirow[b]{2}{*}{ Change in management plan } \\
\hline & & NOF & Trochanteric & Sub-trochanteric & Total & \\
\hline \multirow[t]{6}{*}{ Method } & CS & 31 & 0 & 0 & 31 & 0 \\
\hline & DHS & 39 & 99 & 0 & 138 & 0 \\
\hline & IMN-S & 1 & 111 & 4 & 116 & 0 \\
\hline & IMN-L & 0 & 31 & 29 & 60 & 1 \\
\hline & Other & 0 & 2 & 2 & 4 & 0 \\
\hline & Total & 71 & 243 & 35 & 349 & 1 \\
\hline \multicolumn{2}{|c|}{ Change in management plan } & 0 & 0 & 1 & 1 & \\
\hline
\end{tabular}

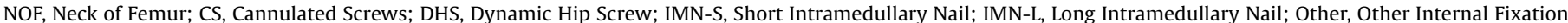

average age of these patients was $83.9 \pm 10.4$ years and $88.2 \%$ were females.

The cost of outpatient clinic visit in our hospital is $€ 140$, and the cost of hip radiographs is $€ 42$ (two views), totaling $€ 182$ per fracture. The total costs of the first outpatient clinic visit in our study population was $€ 63518$, resulting in $€ 31759$ annually in our institution. Our institution's catchment area represents approximately $10 \%$ of the population of Finland.

\section{Discussion}

We found that the first scheduled visit within ten weeks after internal fixation of a proximal femoral fracture leads to no changes in treatment. These follow-ups are scheduled on a routine basis, so the work load of these visits is considerable.

Similar results have been reported for several other fractures. Ghattas et al. [9] noted that after fixation of an acute fracture, the majority of radiographs taken during the first post-operative visit do not alter the patient's treatment plan; they just lead to extra costs for the patient and the health care system [9]. According to Stone et al. [12], routine two-week post-operative radiographs for wrist fracture patients rarely result in changes in patient management [12]. Ovaska et al. [11] recently studied the role of an outpatient visit after an ankle fracture surgery. The results suggested that routine radiographs would not be necessary at an early outpatient visit after ankle fracture surgery. However, $9.8 \%$ of 878 patients required a change in treatment plan post-operatively and only $37 \%$ of these patients had solicited earlier outpatient visit prior to scheduled follow-up visit, therefore it seems reasonable to continue these outpatient visits, since $6.2 \%$ of patients had a change in the treatment plan at the scheduled follow-up visit [11].

Our findings would suggest that the same is not true for PFF patients treated with internal fixation. Fourteen patients $(3.2 \%)$ sought medical care prior to the scheduled follow-up visit whereas only $0.29 \%$ of patients had a change in treatment plan at the scheduled follow-up visit.

This study has some limitations. Patients with acute problems (e.g., infection, acute pain, re-fracture) with the operated PFF are likely to seek medical attention before the scheduled follow-up, in our study this was true for 14 patients with sixteen visits (3.2\%). Thus, these patients do not attend the routine follow-up. This is supported by the low rate of changes in our patients' treatment plans at the scheduled follow-up visit (0.29\%), which is lower than the overall re-operation rate for all PFFs (including hemiarthroplasties): 6.1-6.9\% [13,14]. Also, in none of the scheduled follow-up visits at a special care hospital (Töölö) did the patients present with a wound infection; the overall infection rate in these patients has been reported to be low (0.7\%, 4 year follow-up since surgery) [14]. In our study population patients who attended the first follow-up visit showed no mechanical complications. This is less than the overall mechanical complication rate $(2.7 \%, 4$ year follow-up since surgery) [14] for internally fixated PFFs, but it is likely to be explained by our short follow-up (up to 10 weeks). This emphasizes our goal in this study. We strictly evaluated the changes that took place on the first scheduled post-operative follow-up visit (less than ten weeks after surgery). Our main question was: Is routine follow-up within ten weeks postoperatively necessary in patients who underwent surgery for proximal femoral fracture by internal fixation? The answer is no, the routine follow-up does not lead to changes in the treatment plan. Patients with complications in the operated hip will seek medical attention through an ER and not through a scheduled follow-up visit. The patients lost in the follow-up can potentially modify the results. Our electronic medical records system covers public sector hospitals in southern Finland for approximately 1.2 million inhabitants. The wound infections requiring revision surgery are treated in public hospitals in Finland. Also, the revision surgeries for hip fractures are done in public hospitals. The electronic medical records did not indicate that the patients lost to follow-up were treated in other public hospitals in our hospital district.

To our knowledge, this is the first study to evaluate the necessity of a scheduled post-operative follow-up visit among PFF patients treated with internal fixation. This study evaluated not only the necessity of post-operative radiographs, but also the first scheduled follow-up visit. The results are clinically and socioeconomically notable due to the large number of such surgeries annually.

Our results suggest that orthopaedic surgeons should reassess the need for the first scheduled post-operative follow-up visit (less than ten weeks after surgery) for PFF patients treated with internal fixation. Only a small number of the complications PFF patients experience post-operatively are hip related, and no identified predictors are available for hip-related complications [15]. We suggest increasing post-operative patient guidance in order to teach patients (or to the personal of community-dwellers) the knowledge of when to seek medical attention as well as to encourage patients to contact the operating hospital if they notice any signs of a wound infection or new symptoms in the operated hip. According to our study results, a scheduled follow-up visit within ten weeks of surgery seems unnecessary for PFF patients treated with internal fixation. For this reason, we have omitted now these routine follow-ups in our hospital.

\section{Conclusions}

We recommend orthopaedic surgeons to consider the real need for these routine follow-ups. Given the high incidence of PFFs, discontinuing these follow-up visits would save hospital resources and lower overall health care costs, and - most importantly without risking patient safety. However, more research is needed on the efficacy of follow-up visits scheduled after ten weeks postoperatively. 


\section{References}

[1] Stats OECD, Available at: https://stats.oecd.org, Accessed 12/13, 2016.

[2] Choosing Wisely. Available at: http://www.choosingwisely.org, Accessed 12 13, 2016.

[3] Sittitavornwong S, Waite PD, Holmes JD, Klapow JC. The necessity of routine clinic follow-up visits after third molar removal. J Oral Maxillofac Surg 2005 Sep;63(9):1278-82.

[4] Hacking C, Weinrauch P, Whitehouse SL, Crawford RW, Donnelly WJ. Is there need for routine follow-up after primary total hip arthroplasty? ANZ J Surg 2010;80(October (10)):737-40.

[5] Johnell O, Kanis JA. An estimate of the worldwide prevalence, mortality and disability associated with hip fracture. Osteoporos Int 2004;15(November (11)):897-902.

[6] Gullberg B, Johnell O, Kanis JA. World-wide projections for hip fracture. Osteoporos Int 1997;7(5):407-13.

[7] R Sund, M Juntunen, P Lüthje, T Huusko, M Mäkelä, M Linna, et al. National Institute for Health and Welfare - PERFECT Study. Available at: http://urn.fi/ URN:NBN:fi-fe201204193948, Accessed 12/13, 2016.

[8] AO Foundation. AO Foundation. Available at: https://www2.aofoundation.org/ wps/portal/surgery,Accessed 12/13, 2016.
[9] Ghattas TN, Dart BR, Pollock AG, Hinkin S, Pham A, Jones TL. Effect of initial postoperative visit radiographs on treatment plans. J Bone Joint Surg Am 2013;94(May (9))S1e571 S1.

[10] McDonald MR, Bulka CM, Thakore RV, Obremskey WT, Ehrenfeld JM, Jahangir $\mathrm{AA}$, et al. Ankle radiographs in the early postoperative period: do they matter? J Orthop Trauma 2014;28(September (9)):538-41.

[11] Ovaska MT, Nuutinen T, Madanat R, Makinen TJ, Soderlund T. The role of outpatient visit after operative treatment of ankle fractures. Injury 2016 (September).

[12] Stone JD, Vaccaro LM, Brabender RC, Hess AV. Utility and cost analysis of radiographs taken 2 weeks following plate fixation of distal radius fractures. J Hand Surg Am 2015;40(June (6)):1106-9.

[13] Thakar C, Alsousou J, Hamilton TW, Willett K. The cost and consequences of proximal femoral fractures which require further surgery following initial fixation. J Bone Joint Surg Br 2010;92(December (12)):1669-77.

[14] Tsang ST, Aitken SA, Golay SK, Silverwood RK, Biant LC. When does hip fracture surgery fail? Injury 2014;45(July (7)):1059-65.

[15] Mariconda M, Costa GG, Cerbasi S, Recano P, Aitanti E, Gambacorta M, et al. The determinants of mortality and morbidity during the year following fracture of the hip: a prospective study. Bone Joint J 2015;97-B(March (3)):383-90. 